\title{
ON A THEOREM BY LEON GREENBERG
}

A. W. MASON

1. Introduction. Let $\Gamma$ be the inhomogeneous modular group. $\Gamma$ is the group of all $2 \times 2$ integral matrices of determinant 1 , in which we identify a matrix with its negative. Let $g$ be a normal subgroup of finite index $\mu$ in $\Gamma$. The level of $\mathcal{G}$, as defined in [7], is the minimum positive integral power, $N$, such that $\pm U^{N} \in \mathcal{G}$, where

$$
U=\left[\begin{array}{ll}
1 & 1 \\
0 & 1
\end{array}\right] \text {. }
$$

Any parabolic matrix in $\Gamma$ is one for which the trace is \pm 2 , and $\mathcal{G}$ has a complete system of $t$, say, parabolic representatives, $P_{1}, \cdots$, $P_{t}$, which have the properties that any parabolic element of $g$ is conjugate in $g$ to a power of one of the $P_{i}$ and no nontrivial power of $P_{i}$ is conjugate in to a power of $P_{j}(1 \leqq i \neq j \leqq t) . t$ is called the parabolic class number. It is well known that $\mu=N t$. See [2].

In [1] Greenberg has shown that if $g$ is a normal subgroup of finite index $\mu$ in $\Gamma$ and with parabolic class number $t$, then $\mu \leqq 6 t^{4}$. His proof relies on the use of group extensions and the principal application of his result is to establish a conjecture made by Morris Newman in [6] that there are only finitely many normal subgroups of $\Gamma$ with a given parabolic class number $t$. In this paper we shall show that, if $t>1$, then there exists a positive integer $k$ with $1<k \leqq t$, such that $k \mid N$ and $N \mid k t$. This shows that $\mu=N t \leqq t^{3}$. The restriction that $t>1$ does not affect the generality of the result for, by [6], the only normal subgroups $\mathcal{G}$ of $\Gamma$ for which $t=1$ are $G=\Gamma, \Gamma^{2}, \Gamma^{3}, \Gamma^{\prime}$. We also note that if $G$ is a normal subgroup of $\Gamma$ and $t>1$ then, by [5], $G$ is free.

2. We shall now establish a general property of horocyclic Fuchsian groups. If $L F(2, R)$ is the group of all real linear transformations

$$
T: z \rightarrow \frac{a z+b}{c z+d},
$$

with $a, b, c, d$ real and $a d-b c=1$, which map the upper half complex plane onto itself, then a discontinuous subgroup of $L F(2, R)$ is called a Fuchsian group. In particular the modular group $\Gamma$ and all its subgroups are Fuchsian groups.

Received by the editors March 14, 1969. 
Associated with each Fuchsian group $\mathfrak{F}$ there is a canonical fundamental region $D$ in the upper half plane. See [3]. We assume that the Fuchsian group $\mathcal{F}$ is horocyclic and has a canonical fundamental region which is bounded by a finite even number of segments of noneuclidean lines. Noneuclidean lines are either arcs of circles orthogonal to the real axis or segments of straight lines parallel to the imaginary axis. The sides of $D$ divide off into congruent pairs. This means that there exists an element of $\mathfrak{F}$ which maps one side onto the other side of the pair. It is these elements, which map one member of a congruent pair onto the other, that generate $\mathcal{F}$. By identifying congruent sides of $D$ we can regard the region as a Riemann surface. We can construct $D$ in such a way that each elliptic and parabolic generator of $\mathcal{F}$ maps one side of some adjacent pair onto the other side of that pair, going in an anticlockwise direction around the boundary of $D$. By this construction and by going around the boundary of $D$ in an anticlockwise direction we can obtain a canonical representation for $\mathcal{F}$ in terms of its elliptic, parabolic and hyperbolic generators. We obtain:

$$
\begin{aligned}
& \mathcal{F}=\left\langle E_{1}, \cdots, E_{s}, P_{1}, \cdots, P_{t}, A_{1}, B_{1}, \cdots, A_{g}, B_{g}: E_{i}^{m_{i}}=I\right. \\
& (1 \leqq i \leqq s) \\
& \text { and } \left.\quad E_{1} \cdots E_{s} P_{1} \cdots P_{t} \prod_{i=1}^{g}\left[A_{i}, B_{i}\right]=I\right\rangle
\end{aligned}
$$

where $s$ is the number of elliptic generators $E_{i}$, each of finite integral order $m_{i}(1 \leqq i \leqq s)$, and $t$ is the number of parabolic generators $P_{i}$ of $\mathcal{F}(1 \leqq i \leqq t) . g$ is the genus of the fundamental region, regarded as a Riemann surface, associated with $\mathfrak{F}$.

From the canonical representation we see that $\mathcal{F}$ is free if and only if it has no elliptic elements. Each of the parabolic generators $P_{\boldsymbol{i}}$ of $\mathcal{F}$ is of the form $P_{i}=X_{i} U^{k} X_{i}^{-1}$, where $k$ is a real number and $X_{i} \in L F(2, R)$. the number $k$ is called the amplitude of $P_{\boldsymbol{i}}(1 \leqq i \leqq t)$.

As described previously $D$ is constructed in such a way that each of the $P_{\boldsymbol{i}}$ maps one side of some adjacent pair of sides onto the other side of that pair in an anticlockwise direction. The two sides of such a pair meet at a vertex which must be a fixed point of the parabolic transformation and so must lie on the real axis or be $\infty$.

Remembering that we are going round the boundary of the region in an anticlockwise direction we shall now prove the following theorem concerning the signs of the amplitudes of the $P_{i}(1 \leqq i \leqq t)$. 
THEOREM 1. If $\mathcal{F}$ is a Fuchsian group with the above canonical representation, constructed in the way described, then each of the parabolic generators $P_{i}(1 \leqq i \leqq t)$ has a negative amplitude.

Proof. First of all suppose that one of the $P_{i}$ is of the form $P_{i}=U^{\ell}$; then as the only fixed point of $U^{\ell}$ is $\infty$, this means that $U^{\ell}$ maps two straight lines parallel to the imaginary axis onto one another. As we are going around in an anticlockwise direction this means that $U^{\ell}$ maps such a straight line $\ell_{1}$, say, onto another such straight line $\ell_{2}, \ell_{2}$ lying to the left of $\ell_{1}$, and so $\ell$ must be negative. Suppose now that $P_{i}$ is of the form $P_{i}=A^{-1} U^{-k} A$, with

$$
A=\left[\begin{array}{ll}
a & b \\
c & d
\end{array}\right]
$$

$a, b, c, d$ real, $c \neq 0$ and $a d-b c=1$. The $P_{i}$ maps two circles orthogonal to the real axis onto one another, in an anticlockwise direction, in either of two ways, as depicted in Figures 1 and 2.

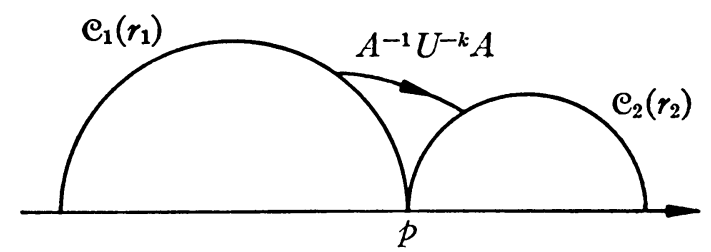

FIGURE 1

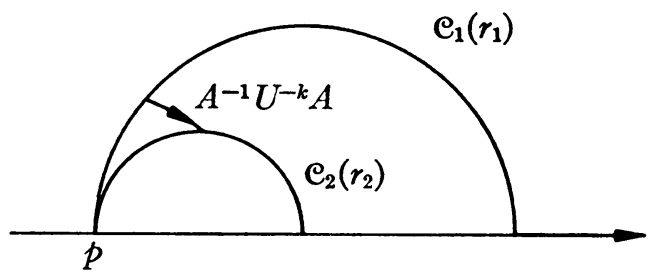

FIGURE 2

In Figure 1 let the circles $\mathfrak{C}_{1}$ and $\mathfrak{C}_{2}$ have radii $r_{1}$ and $r_{2}$, respectively, and let them meet at a point $p$ on the real axis.

As $A^{-1} U^{-k} A$ maps $\mathfrak{C}_{1}$ onto $\mathfrak{C}_{2}, U^{-k}$ maps $A \mathfrak{C}_{1}$ onto $A \mathfrak{C}_{2}$, which must be lines parallel to the imaginary axis, as $A p=\infty$. The equation of $A \mathfrak{e}_{1}$ is $x=A\left(p-2 r_{1}\right)$ and the equation of $A \mathfrak{C}_{2}$ is $x=A\left(p+2 r_{2}\right)$. Since $p=A^{-1} \infty=-d / c$, 


$$
\begin{aligned}
k & =A\left(p-2 r_{1}\right)-A\left(p+2 r_{2}\right) \\
& =\frac{1}{2 c^{2}}\left(\frac{1}{r_{1}}+\frac{1}{r_{2}}\right)>0 .
\end{aligned}
$$

In Figure 2 let $r_{1}$ and $r_{2}$ be the radii of circles $\mathfrak{C}_{1}$ and $\mathfrak{C}_{2}$, respectively, with $r_{1}>r_{2}$. Once again let $p$ be the vertex, noting, as before that $p=A^{-1} \infty=-d / c$.

We find, similarly,

$$
k=\frac{1}{2 c^{2}}\left(\frac{1}{r_{2}}-\frac{1}{r_{1}}\right)>0, \quad \text { as } r_{2}<r_{1} .
$$

This completes the proof of the theorem.

3. We shall now apply Theorem 1 to establish the principle result of the paper. We note that if $\mathcal{F}$ is the modular group $\Gamma$ or if $\mathcal{F}$ is any subgroup of $\Gamma$, the amplitudes of the parabolic generators are integers.

THEOREM 2. If $G$ is a normal subgroup of level $N$ of the modular group $\Gamma$ and if the parabolic class number of $g$ is $t$, where $t>1$, then there exists an integer $k$, with $1<k \leqq t$, such that $k \mid N$ and $N \mid k t$.

Proof. In [6] Newman has shown that there exists a normal subgroup $\mathcal{G}^{*}$ of $\Gamma$, containing $\mathcal{G}$, with level $k$, for some $k$ such that $1<k \leqq t$ and parabolic class number also equal to $t$. (We must have $k>1$, since $\mathrm{S}^{*}=\Gamma$ where $k=1$, implying that $t=1$.)

$\mathcal{G}^{*} / \mathcal{G}$ is cyclic, being generated by the coset $\mathcal{G}^{k}$, so that

$$
\left(\mathcal{G}^{*}\right)^{\prime} \subseteq \mathrm{g} \text {. }
$$

Newman has also shown that $V U^{k} V \equiv U^{k}(\bmod \mathcal{G})$, where

$$
V= \pm\left[\begin{array}{rr}
0 & -1 \\
1 & 0
\end{array}\right]
$$

Since $t>1$, by [5], $\varsigma^{*}$ is free and so has a canonical representation, say

$$
\mathrm{G}^{*}=\left\langle P_{1}, \cdots, P_{t}, A_{1}, B_{1}, \cdots, A_{g}, B_{g}: P_{1} \cdots P_{t} \prod_{i=1}^{g}\left[A_{i}, B_{i}\right]=I\right\rangle .
$$

By Theorem 1, we may choose the parabolic generators $P_{i}$ of $\mathrm{g}^{*}$ such that their amplitudes are all negative. Thus each $P_{i}$ is of the form $P_{i}=X_{i} U^{-k} X_{i}^{-1}$, where $X_{i} \in \Gamma(1 \leqq i \leqq t)$.

Now $V U^{k} \equiv U_{V}^{k}(\bmod \mathcal{G})$, and since $V$ and $U$ generate $\Gamma$,

$$
P_{\boldsymbol{i}} \equiv U^{-k}(\bmod \mathcal{G}) \quad(1 \leqq i \leqq t) .
$$


Since $\mathcal{G}^{*} / \mathcal{G}$ is abelian, the above defining relation for $\mathcal{G}^{*}$ shows that $P_{1} \cdots P_{t} \equiv I(\bmod g)$.

Thus $U^{-k t} \equiv I(\bmod \mathcal{G})$ and therefore $U^{k t} \in \mathcal{G}$, so that $N \mid k t$.

As the level of $\mathrm{G}^{*}$ divides the level of $\mathcal{G}$, we have $k \mid N$.

This completes the proof of the theorem.

COROLLARY 1. There are only finitely many normal subgroups of $\Gamma$ with a given parabolic class number $t$.

Proof. If $t=1$, then $G=\Gamma, \Gamma^{2}, \Gamma^{3}, \Gamma^{\prime}$, as stated in $\S 1$.

If $t>1$, then by Theorem 2 , the index $\mu$ of $\mathcal{G}$ in $\Gamma$ is such that $\mu=N t$ and so $\mu \mid k t^{2}$. Thus $\mu \leqq t^{3}$, as $1<k \leqq t$.

The result follows from this inequality.

Corollary 2. Suppose that $\mathrm{g}$ is a normal subgroup of $\Gamma$ and that $[\Gamma: \mathcal{G}]=\mu$. If $N$ and $t$ are the level and parabolic class number of $\mathrm{G}$, respectively, where $(N, t)=1$ and $t>1$, then $N \leqq t$.

Proof. By Theorem 2, there exists an integer $k$, with $1<k \leqq t$, such that $k \mid N$ and $N \mid k t$. As $(N, t)=1, N \mid k$ and so $N=k \leqq t$.

We note that there are normal subgroups of $\Gamma$ which satisfy the conditions and illustrate the result of Corollary 2 .

For example the principal congruence subgroups $\Gamma(p)$ of $\Gamma$ with level equal to a prime $p$ are such subgroups, since for $p=2, N=2$, $t=3$, while for $p>2, N=p, t=\frac{1}{2}\left(p^{2}-1\right)$.

In conclusion we note that the results of [4] also can be used to derive Greenberg's result in the case when $g$ is a congruence subgroup. For, if $G$ is a congruence group of level $N$, then $G^{*}$ is also a congruence group of level $k$, and, since $G$ is a lattice subgroup of $\mathcal{G}^{*}$, the results of [4] imply that $N \mid 12 k^{2}$, showing that $\mu=N t \leqq 12 t^{3}$.

I should like to express my thanks to Professor Rankin for his many useful hints and suggestions, especially in the proof of Theorem 1.

Addendum. The inequality $N \leqq t^{2}$ has been obtained by R. W. D. Accola in On the number of automorphisms of a closed Riemann surface, Trans. Amer. Math. Soc. 131 (1968), 398-408. Accola's proof in Lemma 5 of this paper uses pure theoretic considerations, the result being obtained by considering the order of a finite group generated by two elements $x, y$, say, such that $x^{2}=y^{2}=(x y)^{N}=1$. It is interesting that this same result can be established by using the fundamental property of the amplitudes of the parabolic generators of a finitely generated horocyclic Fuchsian group.

\section{REFERENCES}

1. L. Greenberg, Note on normal subgroups of the modular group, Proc. Amer. Math. Soc. 17 (1966), 1195-1198. 
2. M. I. Knopp and M. Newman, Congruence subgroups of positive genus of the modular group, Illinois J. Math. 9 (1965), 577-583.

3. A. M. Macbeath, Fuchsian groups, Lectures at Queen's College, Dundee, Scotland, 1961.

4. A. W. Mason, Lattice subgroups of free congruence groups, Glasgow Math. J. 10 (1969), 106-115.

5. M. Newman, Free subgroups and normal subgroups of the modular group, Illinois J. Math. 8 (1964), 262-265.

6. - Classification of normal subgroups of the modular group, Trans. Amer. Math. Soc. 126 (1967), 267-277.

7. K. Wohlfahrt, An extension of F. Klein's level concept, Illinois J. Math. 8 (1964), 529-535.

UNIVERSITY OF GlasGow 